03

\title{
K вопросу определения интегральных характеристик рассеяния
}

\author{
(C) О.Н. Гапоненко \\ Институт ядерных исследований РАН, \\ 117312 Москва, Россия \\ e-mail: olgapone@mail.ru \\ Поступила в редакцию 11.11.2019 г. \\ В окончательной редакции 27.12.2019 г. \\ Принята к публикации 17.01.2020 г.
}

Рассмотрены вопросы, связанные с определением интегральных характеристик индикатрисы рассеяния для сред с остронаправленной в направлении вперед функцией рассеяния. Показано, что в стандартном методе вычисления среднего косинуса угла рассеяния, как правило, не учитывается вклад, важный для рассеяния в направлении малых углов. Приведена методика, позволяющая получить необходимые поправки для интегральных характеристик рассеяния в этом случае.

Ключевые слова: прикладная оптика, поглощение света, рассеяние света, индикатриса рассеяния, средний косинус угла рассеяния.

DOI: $10.21883 /$ OS.2020.05.49321.303-19

Определение характеристик рассеяния естественных сред имеет важное значение как для теории, так и для прикладных аспектов. Так, например, на протяжении долгого времени на озере Байкал ведется сооружение нейтринного телескопа, разные варианты которого (HT-36, НT-72, НТ-144, НT-200, НТ+, Дубна, Baikal GVD) были введены в эксплуатацию и использовались для регистрации нейтрино высоких энергий, см., например, [1-4]. Принцип регистрации в этих установках основан на детектировании света от черенковского излучения, возникающего при распространении высокоэнергетичных заряженных частиц в водной среде озера Байкал.

Известно, что распространение света сопровождается процессами поглощения, в результате которых число фотонов в первичном световом пучке убывает, и процессами рассеяния, в результате которых фотоны изменяют направление распространения. Интенсивность первого процесса принято характеризовать длиной поглощения $\lambda_{a b s}$, которая имеет смысл средней длины между актами поглощения; для второго процесса характеристиками служат длина рассеяни $\lambda_{\text {scat }}$ (средняя длина между актами рассеяния фотонов) и индикатриса (функция) рассеяния $\chi(\theta)$, которая определяет вероятность отклонения фотонов от первоначального направления в элементарный телесный угол в окрестности полярного угла $\theta$ (здесь мы рассматриваем распространенный случай, когда рассеяние не зависти от азимутального угла $\phi$, последнее может не выполняться для кристаллов, однако не вносит принципиально новых моментов в излагаемый в настоящей работе метод).

Для прикладных целей важным является определение оптических характеристик in situ, т. е. непосредственно в месте проведения эксперимента. Так, например, для Байкальского нейтринного телескопа для корректного восстановления траектории регистрируемых частиц необхо- димо знать характеристики поглощения и рассеяния в месте локации оптических детекторов, непосредственно в озере Байкал, на глубине примерно $1300 \mathrm{~m}$, что значительно усложняет измерения по сравнению со случаем лабораторных испытаний [5-7]. Другим фактором, приводящим к значительным сложностям при расчете характеристик рассеянии, является то, что индикатриса рассеяния чистой байкальской воды сильно вытянута в направлении прямо вперед и может меняться на несколько порядков при изменении угла на доли градуса (рис. 1) [7]. Часто вместо индикатрисы рассеяния на практике пользуются интегральными характеристиками, такими, например, как транспортная длина рассеяния

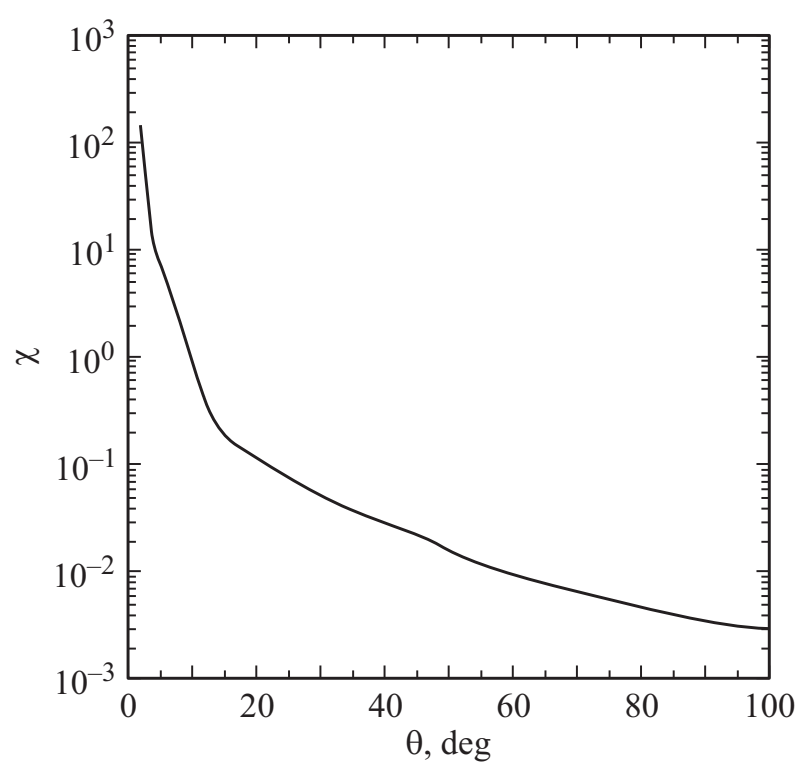

Рис. 1. Индикатриса рассеяния глубинной байкальской воды по данным работы [7]. 
(см., например, [8-11]):

$$
L_{t r}=\frac{\lambda_{\text {scat }}}{1-\langle\cos (\theta)\rangle} .
$$

Здесь $\langle\cos (\theta)\rangle-$ средний косинус угла рассеяния:

$$
\langle\cos (\theta)\rangle=\frac{\int_{0}^{\pi} \cos (\theta) \chi(\theta) \sin (\theta) d \theta}{\int_{0}^{\pi} \chi(\theta) \sin (\theta) d \theta},
$$

интеграл в знаменателе в этом выражении учитывает нормировку экспериментально полученной функции рассеяния на единицу (вероятность рассеяния в полный диапазон углов по всему телесному углу должна равняться 1).

Применение формулы (2) для расчета среднего косинуса угла рассеяния требует знания индикатрисы рассеяния во всем диапазоне углов $\theta$, от 0 и до $\pi$. Как правило, функция рассеяния определяется в эксперименте начиная с некоторого минимального угла $\theta_{\min }$, поэтому формула (2) для экспериментально найденной индикатрисы рассеяния принимает вид

$$
\langle\cos (\theta)\rangle_{\exp }=\frac{\int_{\theta_{\min }}^{\pi} \cos (\theta) \chi(\theta) \sin (\theta) d \theta}{\int_{\theta_{\min }}^{\pi} \chi(\theta) \sin (\theta) d \theta} .
$$

Было найдено (см., например, [6,7]), что в месте нахождения Байкальского нейтринного телескопа для светового излучения с длиной волны $490 \mathrm{~nm}$ $\left.\lambda_{\mathrm{abs}}=20 \mathrm{~m}, \lambda_{\text {scat }}=22.5 \mathrm{~m}, \cos (\theta)\right\rangle_{\text {exp }} \cong 0.95$ здесь минимальный угол составил $\theta_{\min }=2^{\circ}$ в связи с тем, что продвижение в область меньших углов связано с огромными трудностями в измерении сильно меняющейся в этом диапазоне функции рассеяния (рис. 1). Столь близкое к единице значение среднего косинуса рассеяния связано с преобладанием рассеяния в воде естественного водоема на частичках примесей крупных размеров, по-видимому, это в основном органические образования (микроскопические водоросли). К сожалению, для байкальской глубинной воды до сих пор отсутствуют единая теория и подробные данные о распределении этих частичек по их размерам и их прозрачности для различных длин волн видимого света. Для указных значений параметров рассеяния транспортная длина (1) оказывается равной $L_{t r}=450 \mathrm{~m}$. Столь большое значение транспортной длины вовсе не экзотика, а связано с тем, что средний косинус угла рассеяния очень близок к единице для сред, где преобладает рассеяние в направлении прямо вперед, такая ситуация встречается не только при изучении распространения света в естественных водоемах, но и в различных биомедицинских исследованиях [9-11].

Найденное по формуле (3) численное значение для среднего косинуса угла рассеяния не учитывает вклад от области малых углов $\theta<\theta_{\min }$. Вместе с тем несложно показать (рис. 1), что вклад от этой области углов является весьма ощутимым. Например, интеграл от индикатрисы рассеяния по области шириной в $\theta_{\min }$ :

$$
\int_{2^{\circ}}^{4^{\circ}} \chi(0) \sin (\theta) d \theta,
$$

дает вклад более 30\% в интеграл от полного измеренного в эксперименте на рис. 1 диапазона углов. В силу того, что в воде преобладает рассеяние на малые углы, следует ожидать, что вклад от области углов от $0^{\circ}$ до $2^{\circ}$ (т.е. такой же ширины, как и в предыдущем интеграле) будет еще больше. Как правило, для расчета параметров рассеяния в малоугловой области пользуются различными моделями индикатрис, см., например, [12,13]. Однако при таком подходе часто получаются весьма различные результаты в зависимости от выбранной модели, к тому же даже в рамках одной модели применения модельного подхода к водной среде естественного водоема может оказаться малопригодным. Поэтому возникает задача оценки вклада малоугловой области в значение среднего косинуса рассеяния и уточнения найденного по формуле (3) численного значения непосредственно по измеренным в эксперименте данным. Для этого поступим следующим образом: в точной формуле (2) представим числитель и знаменатель в виде двух слагаемых, первое из которых учитывает вклад от области углов от 0 и до $\theta_{\min }$, а второе - от области углов от $\theta_{\min }$ и до $\pi$ :

$$
\begin{aligned}
& \langle\cos (\theta)\rangle= \\
& =\frac{\int_{0}^{\theta_{\min }} \cos (\theta) \chi(\theta) \sin (\theta) d \theta+\int_{\theta_{\min }}^{\pi} \cos (\theta) \chi(\theta) \sin (\theta) d \theta}{\int_{0}^{\theta_{\min }} \chi(\theta) \sin (\theta) d \theta+\int_{\theta_{\min }}^{\pi} \chi(\theta) \sin (\theta) d \theta} .
\end{aligned}
$$

В силу того, что $\theta_{\min }$ обычно мал, в первом интеграле можно заменить $\cos \theta$ его значением для нулевого угла, $\cos (\theta) \approx 1$, это не внесет сколько-нибудь заметной ошибки, точный расчет показывает, что возникающая при этом погрешность во всяком случае много меньше экспериментальных ошибок при измерении индикатрисы $\left(\cos \left(\theta_{\min }\right)=\cos \left(2^{\circ}\right)=0.9994 \approx 1\right)$. Также отметим, что это предположение не является необходимым для излагаемого здесь метода, несложно показать, что можно оставить в первом слагаемом в числителе формулы (4) $\cos (\theta)$ и проводить дальнейшие оценки с этим точным выражением, сделанное здесь упрощение нужно нам лишь для того, чтобы сделать дальнейший вывод более наглядным. С учетом сделанного замечания соотношение (4) принимает 


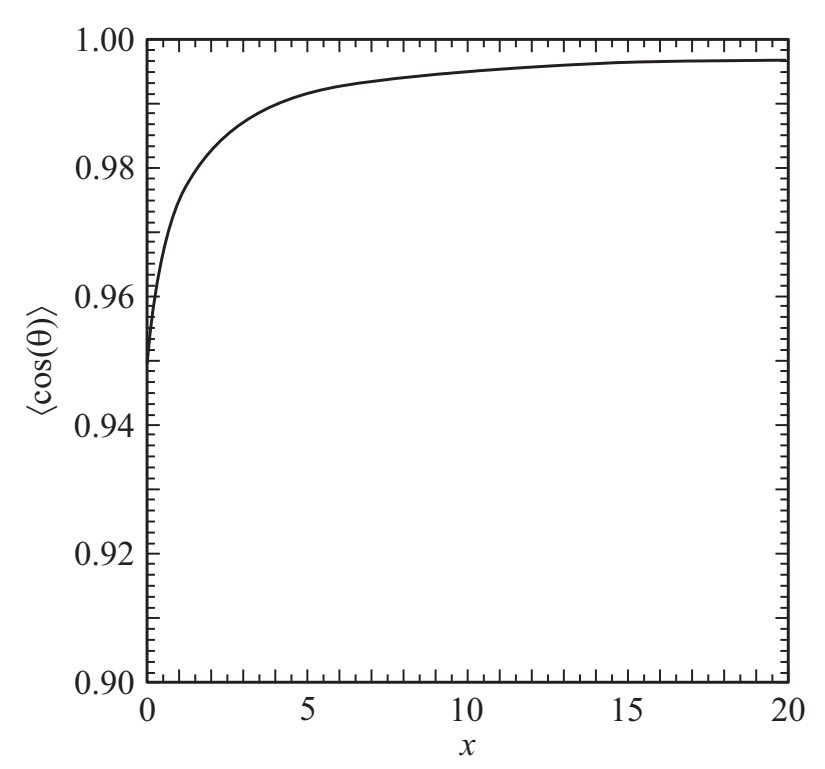

Pис. 2. Средний косинус угла рассеяния как функция параметра $x$, см. формулы (5) и (6) в тексте.

вид

$$
\begin{aligned}
& \langle\cos (\theta)\rangle \approx \frac{\int_{0}^{\theta_{\min }} \chi(\theta) \sin (\theta) d \theta+\int_{\theta_{\min }}^{\pi} \cos (\theta) \chi(\theta) \sin (\theta) d \theta}{\int_{0}^{\theta_{\min }} \chi(\theta) \sin (\theta) d \theta+\int_{\theta_{\min }}^{\pi} \chi(\theta) \sin (\theta) d \theta} \\
& =\frac{\int_{0}^{\pi} \chi(\theta) \sin (\theta) d \theta / \int_{\theta_{\min }}^{\pi} \chi(\theta) \sin (\theta) d \theta}{1+\int_{0}^{\theta_{\min }} \chi(\theta) \sin (\theta) d \theta / \int_{\theta_{\min }}^{\pi} \chi(\theta) \sin (\theta) d \theta} \\
& =\frac{\int_{\theta_{\min }}}{\theta_{\min }} \chi(\theta) \sin (\theta) d \theta / \int_{\theta_{\min }}^{\pi} \chi(\theta) \sin (\theta) d \theta+\langle\cos (\theta)\rangle_{e k s p} \\
& =1-\frac{1+\int_{0}^{\theta_{\min }} \chi(\theta) \sin (\theta) d \theta / \int_{\theta_{\min }}^{\pi} \chi(\theta) \sin (\theta) d \theta}{1+\int_{0}^{\theta_{\min }} \chi(\theta) \sin (\theta) d \theta / \int_{\theta_{\min }}^{\pi} \chi(\theta) \sin (\theta) d \theta}
\end{aligned}
$$

Здесь $\left.\langle\cos (\theta)\rangle\right|_{\exp }-$ найденное по формуле (3) экспериментально значение для среднего косинуса рассеяния. Будем рассматривать отношение $\int_{0}^{\theta_{\min }} \chi(\theta) \sin (\theta) d \theta / \int_{\theta_{\min }}^{\pi} \chi(\theta) \sin (\theta) d \theta \quad$ как некоторый параметр $x$.

Нетрудно видеть, что $x$ может находиться в диапазоне $0 \leq x<\infty$. На рис. 2 показан график уравнения (5) как функция параметра. Точке $x=0$ на графике соот- ветствует ситуация, когда угол $\theta_{\min }$ настолько мал, что вся малоугловая область была измерена в эксперименте, как видно из графика, в этом случае $\left.\langle\cos (\theta)\rangle\right|_{\exp }$ в точности совпадает с ожидаемым $\langle\cos (\theta)\rangle$. Противоположный случай больших значений параметра $x, x \gg 1$, описывает ситуацию, когда преобладающий вклад в средний косинус угла рассеяния происходит от очень малых углов, и, как и следовало ожидать, в этом случае $\langle\cos (\theta)\rangle \rightarrow 1$ (рис. 2). Таким образом, найденный по

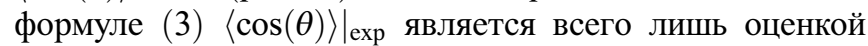
снизу для истинного $\langle\cos (\theta)\rangle$. Как видно из формулы (4) добавление вклада от малоугловой области одновременно увеличивает и числитель, и знаменатель при расчете среднего значения косинуса угла рассеяния. Нужно учесть, что хотя физически очевидно, что малоугловая область содержит все меньшие углы и в связи с этим косинус углов этой области является возрастающей функцией, при расчете этой величины на практике исходят из ненормированной функции рассеяния, полученной в эксперименте по относительному изменению интенсивности света для разных углов регистрации рассеянного света, и кроме возрастания числителя в формуле (4), в ней изменяется и знаменатель. Поэтому полученное на рис. 2 поведение среднего косинуса угла рассеяния как функции параметра $x$ является результатом конкурирующего влияния двух указанных факторов. Чтобы получить более точную оценку среднего косинуса угла рассеяния, необходимо знать значение параметра $x=\int_{0}^{\theta_{\min }} \chi(\theta) \sin (\theta) d \theta / \int_{\theta_{\min }}^{\pi} \chi(\theta) \sin (\theta) d \theta$. Для его оценки поступим следующим образом. Поскольку индикатриса рассеяния является вероятностью рассеяния в телесный угол, а в области малых углов вероятность такого рассеяния становится все больше, то $\chi(\theta)$ является возрастающей функцией. Тогда интеграл $\int_{0}^{\theta_{\min }} \chi(\theta) \sin (\theta) d \theta$ можно оценить снизу, взяв в качестве $\chi(\theta)$ ее минимальное значение в области интегрирования, $\chi\left(\theta_{\min }\right)$, которое известно из эксперимента. Следовательно,

$$
\begin{aligned}
x & =\int_{0}^{\theta_{\min }} \chi(\theta) \sin (\theta) d \theta / \int_{\theta_{\min }}^{\pi} \chi(\theta) \sin (\theta) d \theta \\
& \approx \chi\left(\theta_{\min }\right) \int_{0}^{\theta_{\min }} \sin (\theta) d \theta / \int_{\theta_{\min }}^{\pi} \chi(\theta) \sin (\theta) d \theta .
\end{aligned}
$$

Для экспериментальных данных рис. 1 расчет по формуле (6) дает $x \approx 0.43$. Тогда из формулы (5) получаем следующее уточнение:

$$
\langle\cos (\theta)\rangle \approx 1-\frac{1-0.95}{1+0.43}=0.965
$$

На первый взгляд может показаться, что это уточнение незначительно, и практически не изменяет найденное экспериментальное значение среднего косинуса угла 
рассеяния. Однако это не так. Следует учесть, что чем ближе средний косинус угла рассеяния к единице, тем заметнее становится вклад таких уточнений. Например, для важной при описании времен задержек фотонов величины $L_{t r}$ (см. уравнение (1)), подставляя экспериментальные данные и их уточнение, теперь получим

$$
L_{t r}=\frac{22.5}{1-0.965}=643 \mathrm{~m},
$$

что заметно отличается от использованной ранее в Байкальском нейтринном телескопе транспортной длины $450 \mathrm{~m}$. Выполненные автором расчеты показывают, что найденное по формуле (8) значение транспортной длины рассеяния гораздо лучше описывает распределение времен задержки фотонов в байкальском эксперименте.

\section{Заключение}

Часто на практике вместо полного набора характеристик рассеяния исследователи вынуждены пользоваться более упрощенными величинами, такими, например, как средний косинус угла рассеяния, см., в частности, [8-11]. Такой подход удобен тем, что вместо всей функции рассеяния используется одна интегральная величина, которая, во-первых, менее чувствительная к ошибкам измерения индикатрисы рассеяния и, во-вторых, не требует точного знания функции рассеяния в области малых углов. Однако в сильно анизотропных для рассеяния средах, где преобладает рассеяние света в направлении строго вперед, расчет среднего косинуса угла рассеяния без учета малоугловой области может приводить, как показано в этой работе, к заметной ошибке. Нами предложен подход, который позволяет уточнить значения среднего косинуса угла рассеяния с учетом влияния малоугловой области. Несмотря на простоту, этот подход дает способ получения поправки к экспериментально полученным данным и может быть с успехом применен в различных ситуациях на практике.

\section{Конфликт интересов}

Автор заявляет, что у него нет конфликта интересов.

\section{Список литературы}

[1] Belolaptikov I.A. etal. // Astr. Phys. 1997. V. 7. P. 263.

[2] Aynutdinov V. et al. // Physics of Atomic Nuclei. 2006. V. 69. N 11. P. 1914-1921.

[3] Aynutdinov V. etal. // Astrophys. J. 2008. V. 29. P. 366.

[4] Avrorin A.D. etal. // EPJ Web of Conferences. 2019. V. 207 P. 01003.

[5] Balkanov V.A. etal. // Appl. Opt. 1999. V. 3. P. 68.

[6] Гапоненко О.Н., Миргазов Р.P., Таращанский Б.А. // Оптика атмосферы и океана. 1996. Т. 9. № 08. С. 1069-1076.
[7] Таращанский Б.А., Гапоненко О.Н., Добрынин В.И. // Оптика атмосферы и океана. 1994. Т. 7. № 11-12. C. $1508-1515$.

[8] Исимару A. Распространение и рассеяние волн в случайно-неоднородных средах. М.: Мир, 1981. Т. 1.

[9] O'Sullivan T.D., Cerussi A.E., Cuccia D.J., Tromberg B.J. // J. Biomed. Optics. 2012. V. 17. P. 071 311-(1-14).

[10] Mutyal N., Radosevich A., Gould B., Rogers J.D., Gomes A., Turzhitsky V., Backman V. // Optics Express. 2012. V. 20. P. $19643-19657$.

[11] Зимняков Д.А., Ювченко С.А., Исаева А.А., Исаева Е.А., Ушакова О.В. // Опт. и спектр. 2018. Т. 125. № 5. С. 23.

[12] Копилевич Ю., Кононенко М.Е., Задорожная Е.И. // Оптический журн. 2010. Т. 77. № 10. С. 10-14.

[13] Маньковский В.И. // Оптика атмосферы и океана. 2018. T. 31. № 8. С. 634-639. 